\title{
Development and Application Based on MTS Hybrid Test System
}

\author{
Xinjiang Cai ${ }^{1}$, Shizhu Tian ${ }^{1,2}$ \\ 1. School of Civil Engineering, Suzhou University of Science and Technology, Suzhou 215011, \\ PR China \\ 2. Jiangsu Province Key Laboratory of Structure Engineering, Suzhou University of Science \\ and Technology, Suzhou 215011, PR China \\ E-mail: caixinjiang77@126.com
}

\begin{abstract}
It is meaningful to further explore the hardware and software functions of MTS loading system widely used in building structure test at home and abroad. This paper proposes a kind of seismic pseudodynamic test method based on internal command control of software. Three programs including monitor station, sincycle test and rampcontrol test were developed by VB, and the connection with NetSLab platform was completed via master-slaver network, then the test system was established. A single degree of freedom hybrid test of single span bridge was carried out, and a short column of CFRP reinforced was used to be an experimental element. Secondary load for the partial damaged model was implemented to research the mechanics performance of the specimen. The result confirms that the established system is capable of conducting the pseudo-dynamic hybrid test. The hybrid test demonstrates that the proposed internal command control method is feasible and effective, which can provide reference for the system development and seismic research.
\end{abstract}

Keywords: hybrid test; pseudo-dynamic; MTS system; internal command; bridge column

\section{Introduction}

At present, the structure engineering laboratories at home and abroad have been equipped with MTS electro hydraulic servo loading system which can carry out basic static load test, fatigue test and quasi- static test of civil engineering, but only quasi-static test involves seismic performance of structure, and because of the difficulty in directly input the actual ground motion record, so it cannot reflect the real condition. Shaking table test can reflect the structure seismic performance well, but it is difficult to be comprehensive promoted and applied just because of high cost, limits of table size and bearing capacity. Pseudo dynamic test is also a kind of seismic test methods, and the basic configuration is relatively simple. When using the sub structure technique, it is called the substructure pseudo dynamic test. Most of the laboratories have the reaction wall and actuators, which meet the basic demand of hardware conditions, but there is not a general or commercial software program now, the reason is that pseudo dynamic test is more complicated than other methods. It is not only related to dynamic models and dynamic characteristics of the structure, but also has a close relationship with specimens, configuration of actuators and measuring sensors, especially related to the control system, restricting the development of pseudo dynamic test software. So it is very meaningful to develop the MTS system based on the standard seismic pseudo dynamic test program.

MTS system software comes with a pseudo dynamic test software, sold together with hardware equipment, developed by P. Benson Shing at University of Colorado. This software utilized Hilber, Hughes and Taylor's $\alpha$ method with adaptive variable step size, and could carry out one-dimensional plane pseudo dynamic test. But it doesn't yet obtain general application due to many restrictions [1]. Stephen A. Mahin, University of California at Berkeley, in collaboration with MTS company, established open source framework for experimental setup of control shorted for OpenFresco based on the SCRAMNet reflective memory card technology [2-3]. It is widely used among researchers at home and abroad currently [4]. B.F. Spencer at University of Illinois at urbane Champaign developed UI-SIMCOR platform, and conducted a series of hybrid test by using the unique load and boundary condition boxes named LBCB [5]. ISEE plan launched by the Center for Research on Earthquake Engineering in Chinese Taipei developed a structure test platform named PNSE [6-7]. Wang T studied different test types by using distributed hybrid test platform [8]. Qiu F W at Tsinghua University developed pseudo dynamic test software named TUT based on MTS VC++ library which could conduct a one-dimensional or twodimensional pseudo dynamic test, and substructure with torsion effect was taken into account [9]. It has been applied in a few domestic laboratories. 


\section{Develop program of MTS internal command control}

There are two kinds of methods developing the MTS system, one is hardware system interface and the other is software system interface. Hardware interface of MTS FlexTest GT controller integrates a number of analog and digital controllers. A/D and D/A acquisition boards are used to implement functions of analog input and output, digital input and output, then achieve the operation of control actuator. For the reason of control command signal coming from the external hardware, this method is also known as the external command control method [10-13]. Though this method is simple, the hardware is easily disturbed by environment.

MTS 793 software is the composition of several software packages with powerful functions, including the Function Generator Software, the Basic TestWare Software, the Multi-Purpose Testware Software and the Station Manager Software. As the main control module, the Station Manager Software will send the target command to the actuator and receive the feedback from the actuator via the I/O channel of GT controller during the testing.

Station Manager Software offers a variety of program channel and sends the command to the external servo valve and controller. The program and control channel is used to keep the actuator closed-loop control. When assigned a valve drive resource, this channel type will be selected automatically. An internal command control can be achieved through software programming method to invoke the valve drive, and realize the control of actuator. Using the method above-mentioned, three kinds of programs for MTS software is developed as following.

(1) Monitor station program

Monitor station program shows how to use the data limit detector. It includes command and message severity, Which can demonstrate the connection and security of test system.

As can be seen from figure 1, the program carries out a monitor to the exist station named "Schenck \#1_HSM1_interlock1_wdpexter.cfg", the feedback results is no power, hydraulic interlock and program interlock, and give warning message severity.

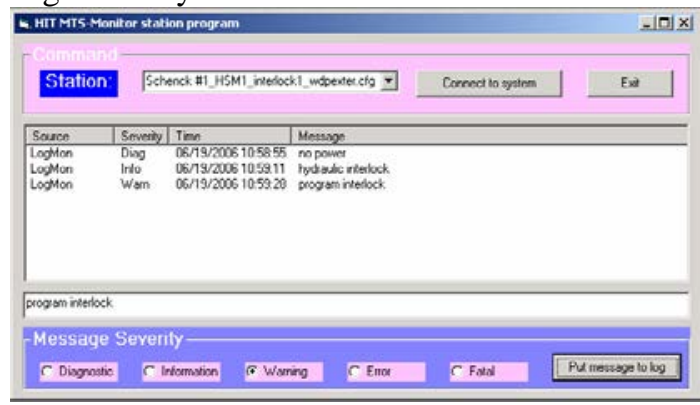

Fig. 1. Monitor station program

(2) Sincycle test program

Sincycle test program includes command, control, interlock, cycle count and feedback shows how to command sine cycles wave and acquiring data at the same time. It can detect the feasibility of test system simply.

As can be seen from figure 2, the program carry out a sine stimulation with the amplitude of $3 \mathrm{~mm}$ and the frequency of $2 \mathrm{~Hz}$. The command wave is preferably identical with the feedback wave in figure 3, which proved that the actuators can meet the demands of simple and slow tests.

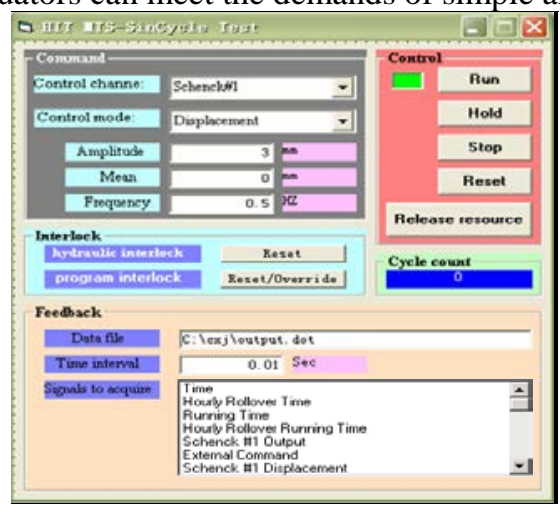

Fig. 2. Sincycle test program

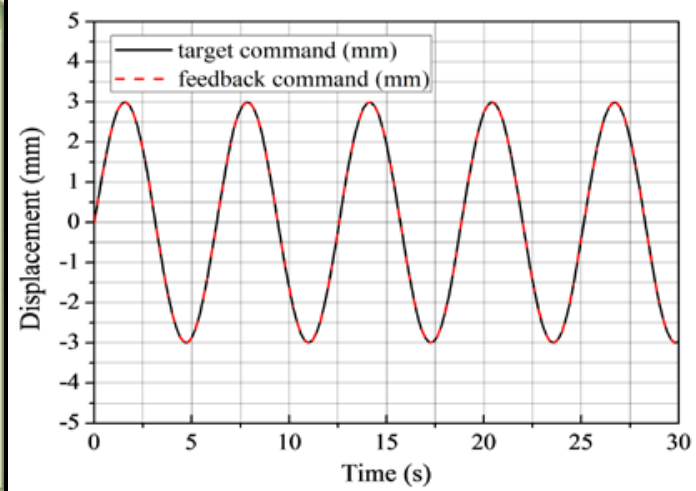

Fig. 3. Comparison of Sin wave loading

(3) Rampcontrol test program

Rampcontrol test program shows how to command a ramp wave and acquiring data at the same time. It includes five parts of function, ramp channel, feedback channel, system command and general command, which 
can demonstrate the two different control modes to create load command and receive feedback command, then release the occurred system resource.

As can be seen from figure 4, a small displacement will produce jitter phenomenon to actuator, a stable response must be waited before data acquisition, and take the average value from many data in order to ensure the accuracy of the results. Taking $0.2 \mathrm{~mm}$ for example, in which the initial position of actuator is set as zero position. The next displacement command is $1 \mathrm{~mm}$, and the ramp program is a step wave , a suspended is set at the second step (i.e. actuator ran to $0.2 \mathrm{~mm}$ ). The program should wait for a smooth response with the actuator, then measure the average value of displacement feedback is $0.2036 \mathrm{~mm}$ and the force feedback is $73.4217 \mathrm{~N}$. Compared with the elastic theoretical calculation of displacement feedback of $0.2000 \mathrm{~mm}$ and force feedback of $71.6000 \mathrm{~N}$, the test results proved that the interface program is running correctly.

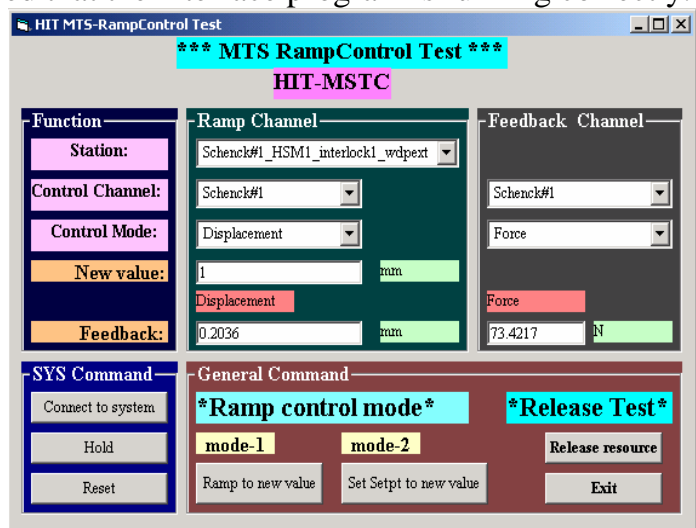

Fig. 4. Rampcontrol test program

\section{Master-Slave network and system integration}

\subsection{Master-slaver network}

MTS FlexTest GT control system includes a system control computer also called Master and a GT controller, both of them are connected by Ethernet to facilitate the exchange of data and transmission faster and more reliable. The control computer is used to operate MTS system software. Hardware interface of GT controller provides a variety of input and output channels. For the reason of security, it is difficult to connect the master computer with internet directly.

In this paper, another additional computer also called Slaver computer is setup, and its function is connecting with internet. on the other hand, Slaver computer is connected to the Master computer through the PC per Station option network options.

The advantage of master-slaver network setup as follows, the Slaver computer has the same function as the Master computer to control single actuator. When a problem happened suddenly, the Master computer can not be affected in any way, and only the Slaver computer can be affected. Data transmission in Master-Slaver network is achieved from the two Hubs installed between two computers. One Hub acts as a hardware sharing network, while another Hub as software sharing network, and different computers need to be set up different network addresses. The diagram of master-slaver as shown in figure 5.

\section{2. system integration}

The integration of monitor station program, sincycle test program and rampcontrol test program with networked test platform named NetSLab is shown as figure 6.
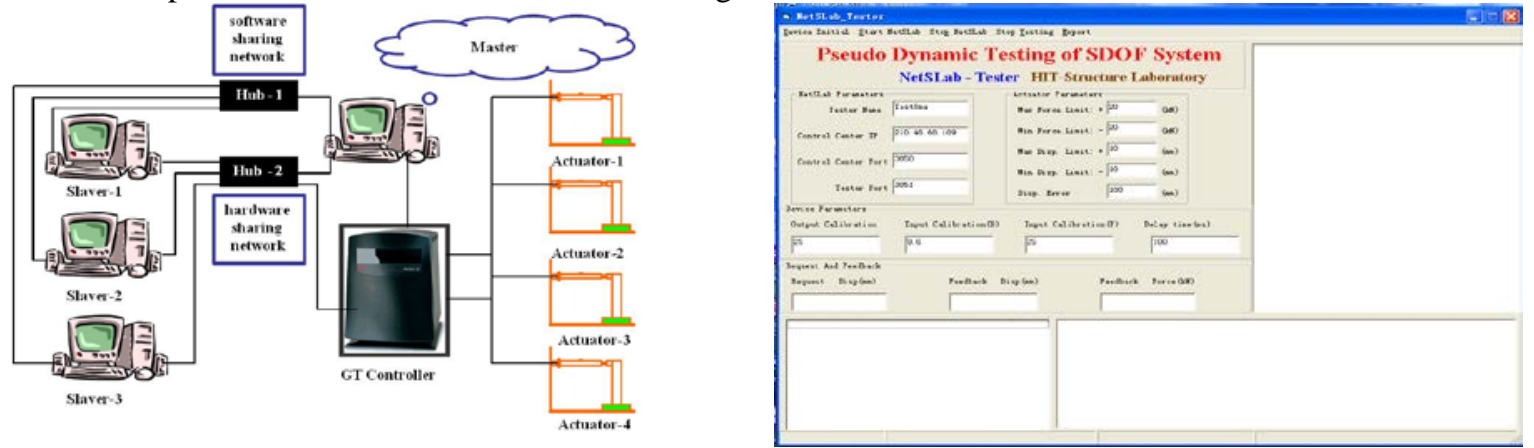

Fig. 5. The frame of Master-Slaver control module Fig. 6. Connection interface between MTS and NetSLab 


\section{Verified hybrid test of a single span bridge}

A single span two columns bridge structure is taken as a model, and assumed to be a single degree of freedom model. Four link test device is applied in hybrid test based on LAN.

Test model is shown in figure 7(b). P1 is the experimental element, and the specimen is a short bridge column of CFRP reinforced shown in figure 7(b). The boundary condition of $\mathrm{P}_{1}$ is fixed with the upper structure. $\mathrm{P}_{2}$ is the numerical element, and its mechanic characters are consistent with $\mathrm{P}_{1}$, while the boundary condition is jointed with the upper structure. The restoring force model is taken as bilinear hysteretic model. A modified CDNewmark combined numerical integral algorithm is established based on the parameter modification of traditional CD-Newmark algorithm. The overall structure conducts the test in terms of the model to simplify the analysis.

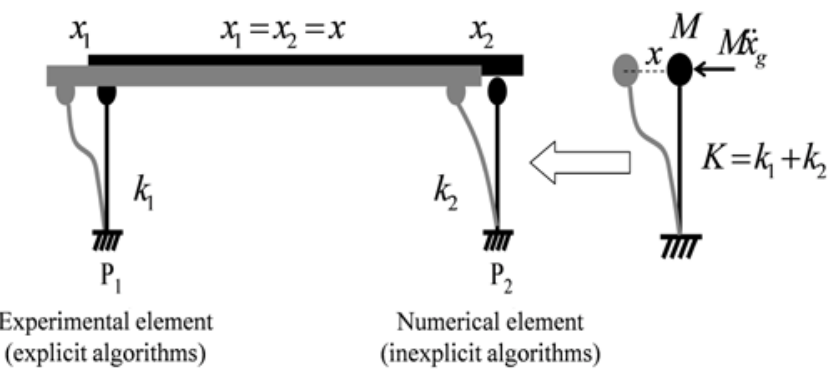

(a) Test modle

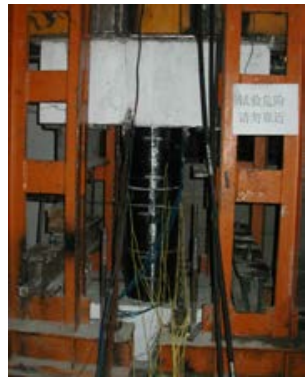

(b) Specimen

Fig. 7. Model of single span two columns bridge

Before the test, the lateral stiffness of experimental elemental is measured as $80000 \mathrm{kN} / \mathrm{m}$, the numerical elemental is also taken as $80000 \mathrm{kN} / \mathrm{m}$. The frequency of whole model is assumed as $2 \mathrm{~Hz}$, and then the calculated mass is $5.1 \times 10^{5} \mathrm{~kg}$, the damping ratio is 0.05 .

EL-Centro earthquake acceleration record is input in the test, and the peak value of acceleration is $0.035 \mathrm{~g}$ and $0.07 \mathrm{~g}$. The bridge column model could be partially damaged and reloaded through adjusting the PGA.

Displacement control method is used in the test to solve the problem of specimen large displacement response. The limited displacement of the actuator is set to $\pm 40 \mathrm{~mm}$ and the limited feedback restoring force is set to \pm 300 $\mathrm{kN}$. Displacement increment in each step is set to $0.05 \mathrm{~mm}$, loading time is $100 \mathrm{~ms}$. Waiting time for a smooth response of an actuator is set to $200 \mathrm{~ms}$ under $0.035 \mathrm{~g}$ and $0.07 \mathrm{~g}$ of partial damage, while set to $1000 \mathrm{~ms}$ under $0.07 \mathrm{~g}$ of secondary load.

Figure 8(a) shows the program running interface under the peak ground acceleration $0.035 \mathrm{~g}$. It can be seen from the figure, the model is linear elastic state due to the small displacement reaction. For the reason of the error limit is set to $0.1 \mathrm{~mm}$, the target displacement is preferably identical with the feedback displacement. The average time of a single step is $26 \mathrm{~s}$.

Figure 8 (b) shows the program running interface under the peak ground acceleration $0.07 \mathrm{~g}$. It can be seen from the figure, the displacement reaction has reached to $15 \mathrm{~mm}$, and the model is already nonlinear plastic state. There are many cracks on the top tensile area of the column. The model has been damaged to a certain extent, and the bearing capacity of column tends to be stable. For the reason of the error limit set to $0.15 \mathrm{~mm}$, the target displacement is a little different from the feedback displacement. The average time of a single step is extended to $26 \mathrm{~s}$.

A further secondary load is imposed to the partial damaged model and the peak ground acceleration is still $0.07 \mathrm{~g}$. The lateral stiffness of model is re-measured to $27500 \mathrm{kN} / \mathrm{m}$ before the test. The displacement error limit is still set to $0.15 \mathrm{~mm}$, but the delay time is increased comparing with the partial damaged work condition of $0.07 \mathrm{~g}$. The target displacement is basically identical with the feedback displacement. The test achieves a better control accuracy, and the average time reduces to single-step 23s. Failure of the bridge volumn is shown in figure 9 . 


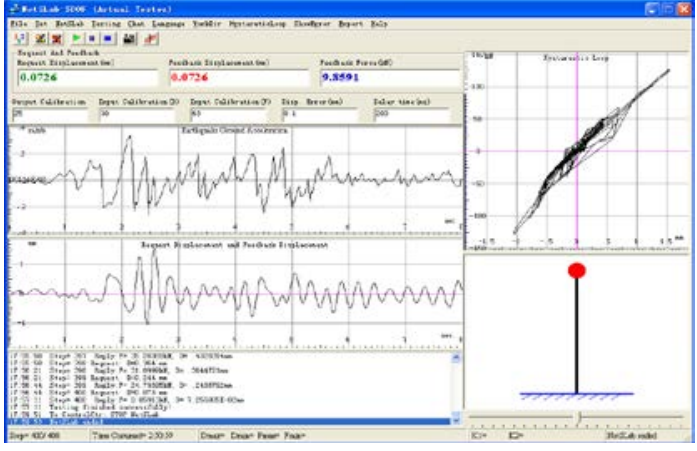

(a) PGA of $0.035 \mathrm{~g}$

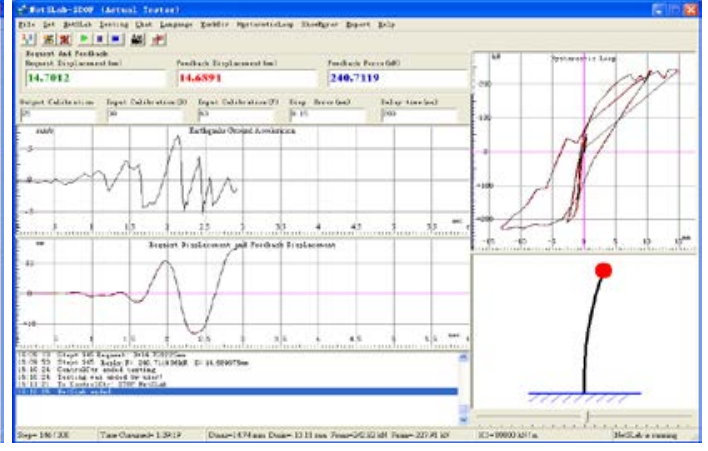

(b) Partially damage under PGA of $0.07 \mathrm{~g}$

Fig. 8. Program running interface

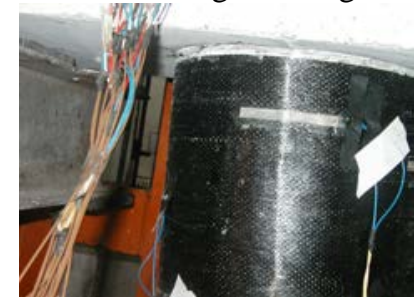

(a) Column cap

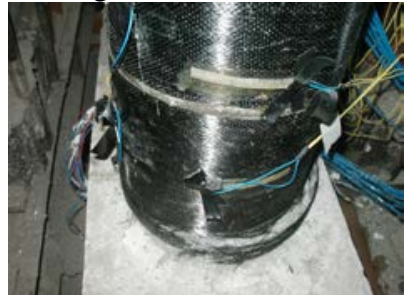

(b) Column base

Fig. 9. Failure of the bridge column

\section{Conclusions}

A relevant software development was carried out based on MTS electro hydraulic servo loading system widely used in building structure test. The pseudo dynamic test system developed in this paper can run smoothly, and has a certain accuracy and stability.

Compared with the external command control, internal command control can make full use of the function of MTS system, avoiding the test error caused by the external hardware.

The load system in this research displays a perfect loading affect when the displacement error limit of $0.15 \mathrm{~mm}$ and delay time of $1000 \mathrm{~ms}$. The single degree of freedom short bridge column test can verify the method and the system in this paper can be applied to seismic pseudo dynamic test.

Compared with the quasi-static test, the pseudo dynamic test can better reflect the real mechanical properties of complex parts of the structure.

\section{Acknowledgements}

This research was supported by the Natural Science Foundation of China $(51778395,51308368)$ and Natural Science Foundation of Jiangsu Province (BK20171221).

\section{References}

[1] MTS Systems Corporation. FlexTest ${ }^{\mathrm{TM}}$ II m program for pseudo-dynamic testing. Eden prairie, Minnesota, 1999.

[2] Takahashi Y., Fenves G.L., Software framework for distributed experimental-computational simulation of structural systems [J]. Earthquake Engineering and Structural Dynamics, 2006, 35(3): 267-394.

[3] Schellenberg A.H., Mahin S.A., Fenves G.L., Advanced implementation of hybrid simulation [R]. University of California, Berkeley, 2009.

[4] Wang Q., Feng F., Zhang F., et al, Research on substructure pseudo-dynamic test with finite element software abaqus [J]. China Civil Engineering Journal, 43 (Supplement): 2010, 515-519. (in Chinese)

[5] Mahmoud H.N., Elnashai A.S., Spencer B.F., et al, Hybrid simulation for earthquake response of semirigid partial-strength steel frames [J]. Journal of structural engineering, 2013, 139: 1134-1148.

[6] Yang Y.S., Hsieh S.H., Tsai K.C., et al., ISEE: Internet-based simulation for earthquake engineering Part I: database approach[J]. Earthquake Engineering and Structural Dynamics, 2007, 36(12): 2291-2306. 
[7] Wang K.J., Tsai K.C., Wang S.J., et al, ISEE: Internet-based simulation for earthquake engineering Part II: the application protocol approach[J]. Earthquake Engineering and Structural Dynamics, 2007, 36(12): 2307-2323.

[8] Wang T., Mosqueda G., Jacobsen A., et al, Simulation of the collapse behavior of structure with distributed hybrid test system [J]. Journal of Disaster Prevention and Mitigation Engineering, 30 (Supplement): 2010, 65-69.

[9] Qiu F.W., Pan P., Qian J.R., et al, Development and application of pseudo-dynamic testing software [J]. Journal of Building Structure, 2000, 21(5): 22-32. (in Chinese)

[10] Xiao Y., Guo Y.R., Fan Y.L., et al, Networked structural laboratories (NetSLab) principles, methods and applications [m]. Beijing: Science Press, 2011. (in Chinese)

[11] Cai X.J., Tian S.Z., Wang D.P., et al. Networked collaborative pseudo-dynamic testing of a multi-span bridge based on NetSLab [J]. Earthquake Engineering and Engineering Vibration. 2009, 8(3):387-397.

[12] Cai X.J., Gong M.H., Jia H.X., et al. Seismic quasi-static test and hybrid test on +-shaped section solid-web steel reinforced concrete columns [J]. Journal of Building Structures. 2016, 37(5):146-154. (in Chinese)

[13] Cai X.J., Jia H.X., Gong M.H., et al, Seismic hybrid simulation of steel reinforced concrete frame with special-shaped columns based on singlecolumn substructure [J]. Journal of Building Structures. 2017, 38(4):35-44. (in Chinese) 